\title{
Cost-effectiveness analysis of comprehensive intervention programs to control blood glucose in overweight and obese type 2 diabetes mellitus patients based on a real-world setting: Markov modeling
}

\author{
Hui Wang", Mengyang Wang", Jiao Wang, Hongwei Liu, Rui Lu, Tongqing Duan, Xiaowen Gong, \\ Siyuan Feng, Zhuang Cui, Yuanyuan Liu, Changping Li, Jun Ma \\ Department of Health Statistics, College of Public Health, Tianjin Medical University, Tianjin 300070, China \\ Contributions: Conception and design: H Wang, M Wang; (II) Administrative support: Y Liu; (III) Provision of study materials or patients: J Ma; (IV) \\ Collection and assembly of data: R Lu, T Duan, X Gong, S Feng, H Liu; (V) Data analysis and interpretation: H Wang, J Wang; (VI) Manuscript \\ writing: All authors; (VII) Final approval of manuscript: All authors. \\ \#These authors contributed equally to this work. \\ Correspondence to: Changping Li; Jun Ma. Department of Health Statistics, College of Public Health, Tianjin Medical University, No. 22 Qixiangtai \\ Road, Heping District, Tianjin 300070, China. Email: lichangping@tmu.edu.cn; junma@tmu.edu.cn.
}

\begin{abstract}
Background: Blood glucose control management in overweight and obese diabetic patients poses heavy public health and economic burdens on the health system. This study aimed to evaluate the short-term costeffectiveness of a comprehensive intervention program for blood glucose management in different groups using a Markov model.

Methods: Based on real-world data, a Markov model was developed to calculate the cost per qualityadjusted life-year (QALY) gained. The division of Markov states was in accordance with clinical practice. A three-month cycle length and a 5-year time horizon were applied. A 3\% discounting rate was applied for both the costs and utilities.

Results: The incremental cost-effectiveness ratios (ICER) was more favorable for the male group than the female group, with an associated ICER of $104 \mathrm{~K} \mathrm{RMB}$ per QALY gained. Compared with the younger group, the incremental gain of the middle-aged group was -0.062 QALY, and the incremental cost was -3,198.64 RMB; meanwhile, the incremental gain of the elderly group was -0.176 QALY, and the incremental cost was 4,485.746 RMB. The sensitivity analysis showed that the ICER is sensitive to the costs of this program and less sensitive to the discounting rate and the time horizon.

Conclusions: The comprehensive intervention program for blood glucose management of overweight and obese patients with diabetes is cost-effective for the middle-aged male group and elderly female group, respectively. Moreover, the male group was more favorable than the female group if three times the gross domestic product (GDP) per capita was adopted as the maximum willingness to pay (WTP) for a QALY in China.
\end{abstract}

Keywords: Cost-effectiveness analysis; Markov model; type 2 diabetes; overweight; obesity; comprehensive intervention program

Submitted Aug 02, 2019. Accepted for publication Sep 30, 2019.

doi: $10.21037 /$ atm.2019.10.38

View this article at: http://dx.doi.org/10.21037/atm.2019.10.38 


\section{Introduction}

According to a research conducted by the First Hospital of Jilin University, the prevalence of adult obesity in northeastern China has reached $37.71 \%$ (1), mirroring an increase in the prevalence of diabetes in overweight and obese people. The prevalence of diabetes in overweight and obese people in China was $12.8 \%$ and $18.5 \%$, respectively (2). Although the cause of diabetes is complicated, being obese and overweight are independent risk factors for diabetes (3). China is being confronted with its most serious diabetes epidemic; there were 114.4 million people with diabetes from 20 to 79 years old in 2017, which was the highest worldwide (4). With the rise in the prevalence of risk factors for type 2 diabetes mellitus (T2DM), including a population being increasingly overweight or obese, and older, the number of individuals with diabetes could be as high as 119.8 million by 2045 (4). Among all types of diabetes, T2DM accounts for around $90 \%$, making it the most common cause of diabetes $(5,6)$. For those who have been diagnosed with type 2 diabetes, the prolonged poor management of diabetes increases the risk of developing serious complications, which is associated with an on average ten-year-shorter life span $(7,8)$. Except for the lower quality of life due to T2DM-related complications, T2DM also places an economic burden on healthcare systems, individuals with T2DM, and their families. In China, the diabetes-related healthcare cost reached US\$ 110 billion in 2017 , accounting for $14.9 \%$ of the global healthcare expenditure on diabetes (4). Moreover, among patients with end-stage renal disease or other diseases, the expenditure of healthcare for individuals with T2DM recently reached up to 3 to 4 times that of those without T2DM (9).

Management of T2DM through blood glucose control and risk factor modification can help prevent and slow the progression of complications (10). The UK-based trials, UKPDS 34 and 51, demonstrated that, for overweight and obese type 2 diabetic patients, intensive blood-glucose control with metformin is cost-effective and extends their life expectancy when compared with the usual care $(11,12)$. However, the results of these studies are essentially different from those in China (13). Currently, individuals with T2DM do not only undergo an oral antidiabetic drug (OAD)-based regimen, but lifestyle intervention is another indispensable component of the therapeutic approach in T2DM, especially for overweight and obese patients (14). Several economic evaluation studies, focusing on a prediabetic population, have also demonstrated that lifestyle intervention is cost-effective for preventing diabetes progression (15-17). Unfortunately, there have been no cost-effectiveness studies conducted which focus on strengthening blood glucose management through comprehensive lifestyle intervention in overweight and obese patients diagnosed with T2DM. Given the increasing health and economic burden on patients and health systems, diabetes management is a key issue for policy-makers. The cost-effectiveness and generalizability of this kind of comprehensive lifestyle intervention have not been analyzed within on the provincial level.

For those overweight and obese diabetic patients enrolled in the Cities Changing Diabetes (CCD) program, the objective of this study was to use the Markov model to assess the cost-effectiveness within the comprehensive intervention program in different age and gender groups.

\section{Methods}

\section{Programs and participants}

The CCD in Tianjin Program was launched in April 2018. It is a comprehensive intervention program aimed at guiding overweight and obese T2DM patients to control their blood sugar and change their lifestyle. The program manages the recruited participants through the "Type 2 Diabetes Care Workstation" established by 47 hospitals in accordance with uniform standards. Patients were recruited from 47 workstations as outpatients eligible for the program from April 2018 to April 2019. The enrolled patients were followed up monthly for one year. These 47 work stations are responsible for drug guidance and blood glucose [including fasting plasma glucose (FPG), postprandial $2 \mathrm{~h}$ plasma glucose (2 h PG), and hemoglobin A1c (HbA1c)] monitoring, on-site questionnaire survey, regular monthly follow-up calls, and health education. The management process includes the first face-to-face education program, and the comprehensive management of monthly follow-ups after enrollment, which includes two aspects. The first includes individualized drug regimens and guidance being used for patients with different clinical characteristics and each patient being told about the health issues associated with obesity to make them more aware of their health status. The second is conducting appropriate lifestyle interventions, including physical exercise (to guide individualized exercise patterns, exercise intensity and time, and regular telephone follow-up supervision to conduct body mass measurement and recording), and diet guidance 


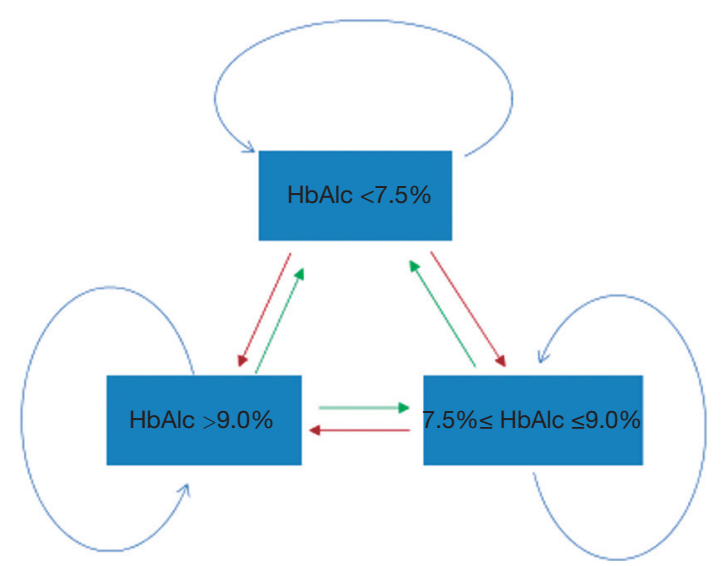

Figure 1 State-transition diagram.

depending on the patient's situation. The CCD program determines the total calorie of the daily diet (calculating the daily food exchange, rationally distributing three meals a day), and the direction of counseling. A survey is used and includes the following items: the clinic's use of the projectmade questionnaire to collect general information (sex, age, smoking, drinking, living conditions, etc.) and diabetesrelated data (diabetes, disease history, family history, exercise, and diet). For the physical examination, the patient is required to take off heavy clothing and be measured for height, body mass, waist circumference, and other indicators. The average value after three measurements is taken as the final measurement result.

Participants, both women and men were, aged 18 to 70 years old, with a BMI of $24 \mathrm{~kg} / \mathrm{m}^{2}$ or higher, having lived in Tianjin for at least three years or longer, diagnosed with type 2 diabetes were selected from 47 hospitals in Tianjin. All of them have signed informed consent before participation in the study. T2DM patients with serious complications or comorbidities were excluded. The protocol of this study was approved by the Institutional Review Board of Tianjin Medical University. A total of 2,145 patients were enrolled in the one-year patient recruitment period, but by the time of our analysis conducted in December 2018, a total of 987 patients who completed a three-month comprehensive intervention were finally enrolled in our final study. According to the instructions of the Working Group of Obesity in China, adults weighing $24 \mathrm{~kg} / \mathrm{m}^{2} \leq \mathrm{BMI}<28 \mathrm{~kg} / \mathrm{m}^{2}$ are overweight, and those whose BMI is more than $28 \mathrm{~kg} / \mathrm{m}^{2}$ are obese.

\section{Markov model}

A Markov chain model, which is the simplest Markov model, was used to estimate the cost-effectiveness of this comprehensive intervention program. The Markov model is a process that models random events that occur over time and has been increasingly applied to areas in health economic research in recent years, such as screening for disease, decision analysis of clinical interventions, pharmacoeconomic evaluation, and so on (18-21). The principle is to divide the natural course of the disease into several different health states (Markov state) and to simulate the development of the disease according to the transition probability of each state in a certain period of time (Markov cycle). This information is combined with the health utility values and the public health resource consumption of each state to estimate the outcome and the cost of disease progression through multiple iterations (22). The Markov model of this study consisted of three health states. These states represent different conditions of blood glucose management in diabetic patients. The input parameters for these states were obtained from the mean of the actual population participating in the program. The Markov model is characterized by a population with mean values for risk factors that are adjusted by the intervention effect. A participant at stage 1 (HbA1c $<7.5 \%$ ), after a 3 -month cycle, can remain in this stage, or enter stage $2(7.5 \% \leq \mathrm{HbA} 1 \mathrm{c}$ $\leq 9.0 \%$ ), or stage 3 (HbA1c $>9.0 \%$ ) (23). For regression, a participant in stage 3 (HbAlc $>9.0 \%$ ) can remain in this stage, or enter stage $2(7.5 \% \leq \mathrm{HbA} 1 \mathrm{c} \leq 9.0 \%)$, or enter stage 1 ( $\mathrm{HbA} 1 \mathrm{c}<7.5 \%)$. This change is possible because comprehensive interventions may have an impact on this transition. The transition process is shown in Figure 1.

HbA1c is an important indicator of long-term glucose management in diabetic patients, and this indicator is not clinically significant until three months after the intervention. Thus, a three-month cycle was adopted in our analysis. When an entire program is completed, there is no longer any monthly follow-up, and it is difficult for patients to maintain their previous high standard for blood glucose management. Moreover, the intervention effect of many lifestyle intervention programs gradually weakens within 3 to 10 years after the end of the intervention (15). After discussion with clinical experts, we adopted a fiveyear horizon from a conservative perspective. For cost estimation, the societal perspective was applied. 
Table 1 The transition probability of different age and sex

\begin{tabular}{|c|c|c|c|}
\hline & \multicolumn{3}{|c|}{ To } \\
\hline \multicolumn{4}{|l|}{ Sex: male, female } \\
\hline From $\mathrm{HbA} 1 \mathrm{c}<7.5 \%$ & $0.87,0.83$ & $0.11,0.12$ & $0.02,0.05$ \\
\hline From $7.5 \% \leq \mathrm{HbA} 1 \mathrm{c} \leq 9.0 \%$ & $0.81,0.81$ & $0.19,0.19$ & $0.00,0.00$ \\
\hline \multicolumn{4}{|c|}{ Age: $<45$ years, $45-60$ years, $>60$ years } \\
\hline From $\mathrm{HbA} 1 \mathrm{c}<7.5 \%$ & $0.89,0.83,0.87$ & $0.09,0.15,0.08$ & $0.02,0.02,0.06$ \\
\hline From $7.5 \% \leq \mathrm{HbA} 1 \mathrm{c} \leq 9.0 \%$ & $0.82,0.81,0.82$ & $0.17,0.17,0.17$ & $0.02,0.02,0.02$ \\
\hline From $\mathrm{HbA} 1 \mathrm{c}>9.0 \%$ & $0.78,0.11,0.55$ & $0.15,0.23,0.36$ & $0.06,0.66,0.09$ \\
\hline
\end{tabular}

\section{Input parameters}

\section{Transition probabilities}

The conditional probability of a given stage moving to another stage after a certain time is expressed by transition probabilities. In Figure 1, the arrows indicate whole possible transitions between the states within the span of three months. The transfer probability of this study was extracted from the study of the CCD intervention scheme (Table 1). Stages of diabetic patients in this study were distinguished based on the level of HbAlc at first examination, which is used for the monitoring of the degree of blood glucose control in the individuals with diabetes. At the initial stage of the analysis, the participants were stratified by gender and age. Participants were divided into three age groups, including the younger group (under 45 years), the middleaged group (between 45 and 60 years), and the elderly group (above 60 years).

\section{Utilities}

The generic health status and health-related quality of life (HRQoL) were expressed by health utility weights (HUWs). HUWs are valued between 0 and 1 according to how the individuals perceive his or her health status. An individual with a utility of one regards her or his status as "perfect health", while someone with a utility of zero regards her or his status as "death". Quality-adjusted lifeyears (QALYs) were calculated by multiplying utilities with the years lived in that state. The health utilities in this study were based on another study population from March 2014 to June 2014 (24). Based on the severity of complications and comorbidities in that group of type 2 diabetic patients, the health utilities were calculated for each health condition. The health utilities were derived from the EQ-5D instrument and translated to EQ-5D values. In this study, each patient was assigned a health utility based on their conditions of comorbidities and complications. The health utility value in each state is the mean of the health utility value of all participants in its state. With worsened complications or comorbidities present, the health utility for one person decreases.

\section{Costs}

All costs are expressed in RMB and base on 2018 prices. Costs for T2DM patient management consist of direct medical costs and indirect medical costs (Table S1). The direct medical cost was divided into screening fee, measurement of blood lipids, glycated hemoglobin, glucose tolerance, and drug costs. According to each patient's physical conditions, this program implements individualized drug regimen management based on the medication instructions from selected experts. The drugs consist of all kinds of diabetes medication, and the costs of drugs are derived from data from hospitals in Tianjin. The average 3 -month medication costs of each participant were estimated based on the actual medication and examination of patients. Other costs like the telephone hotline fee and transportations costs were also taken into account. Participants' transportation costs to hospitals were estimated by using an average 5 -km driving distance multiplied by the general mileage allowance for Tianjin, China. Project staff follow-up time included 3 minutes per month of telephone counseling per participant. Costs for the telephone hotline were estimated 
Table 2 The costs and utilities of different ages and sexes

\begin{tabular}{lcc}
\hline Parameter 2 & Deterministic value & $95 \% \mathrm{Cl}$ \\
\hline Cost (RMB) & & \\
Male & $3,196.80$ & $(2,259.60-4,421.24)$ \\
Female & $3,388.46$ & $(2,350.80-4,597.68)$ \\
$<45$ years & $3,231.48$ & $(2,371.80-4,246.80)$ \\
$45-60$ years & $3,264.30$ & $(1,998.36-4,514.77)$ \\
$>60$ years & $3,237.55$ & $(2,259.60-4,616.89)$ \\
Utility & & \\
Man & 0.859 & $(0.815-0.859)$ \\
Woman & 0.859 & $(0.764-0.859)$ \\
$<45$ years & 0.859 & $(0.815-0.859)$ \\
$45-60$ years & 0.859 & $(0.815-0.859)$ \\
$>60$ years & 0.815 & $(0.764-0.859)$ \\
\hline
\end{tabular}

according to the tariff standard of the local operators. An annual discount rate of $3 \%$ is applied for both the costs and utilities.

Both utilities and costs were calculated by using the data from the program according to the methods mentioned above. The utilities and costs of different age and gender groups are summarized below (Table 2). Three months' average cost per person was generally adopted in the model.

\section{Other parameters}

\section{Willingness to pay (WTP)}

In a developing country, the maximum price of one year of a healthy life is around threefold the annual earning per capita (12). The gross domestic product (GDP) per capita in Tianjin is $118,944 \mathrm{RMB}(17,324 \mathrm{US} \$)$. The maximum WTP for a QALY gained in this program of about $356.8 \mathrm{~K}$ $\mathrm{RMB}$ was estimated based on the above approach.

\section{Sensitivity analyses}

One-way sensitive analyses were performed in three ways to determine the main factors that affect the incremental costeffectiveness ratio (ICER). The costs of states were varied by plus and minus $10 \%$ to investigate the influence of the variance on costs estimates. The time of the intervention showing effect was doubled from 5 to 10 years to investigate whether this lower assumed duration of effect influenced the results. Moreover, the annual discount rate was applied from $0 \%$ to $6 \%$ to investigate whether this parameter influenced the model results. Both cost-effectiveness analysis and sensitivity analysis were performed using TreeAge Pro Healthcare 2018 (Tree Age Software, Williamstown, WA, USA).

\section{Results}

\section{Baseline demographics}

The study included 987 patients with type 2 diabetes that were overweight or obese who had completed a 3-month comprehensive intervention. There were 606 males (61.4\%) and 381 females (38.6\%). There were 249 overweight patients $(25.2 \%)$ and 738 obese patients $(74.8 \%)$. The number of patients with central obesity was $872(88.3 \%)$. The mean values of fasting blood glucose, $2 \mathrm{~h}$ postprandial blood glucose, and $\mathrm{HbA1c}$ were $8.6 \mathrm{mmol} / \mathrm{L}, 11.7 \mathrm{mmol} / \mathrm{L}$, and $8.5 \%$, respectively (Table 3).

\section{Base case}

The costs and health outcomes of the intervention for different gender and age groups in the included participants over five years are summarized in Table 4.

Among all age groups, the middle-aged group had the lowest cost of intervention, the elderly group had the highest cost, while the youngest group gained the highest QALYs. Compared with the youngest group, the incremental gain of the middle-aged group was -0.062 QALY, and the incremental cost was -3,198.64 RMB, while the incremental gain of the elderly group was -0.176 QALY, and the incremental cost was 4,485.746 RMB.

At the end of this period, the difference in QALYs between the female group and the male group was relatively small. Women have lower costs compared to men. While the ICER is positive for the male group compared to the female group, the comprehensive intervention resulted in an incremental gain of 0.054 QALY, and the incremental cost was 5,618.39 RMB in the male group.

The effects and costs of the intervention program in each subgroup during five years of follow-up are summarized in Table 5. For the middle-aged women and elderly age women, the comprehensive intervention led to cost-savings in comparison to the younger age women. This means the intervention will save money in the elderly female groups, as fewer people will transition to a higher-risk health state.

In the male group, the ICER was negative for the 
Page 6 of 10

Table 3 Baseline demographics

\begin{tabular}{|c|c|}
\hline Characteristics & $\mathrm{n}(\%)$ or mean (IQR) \\
\hline \multicolumn{2}{|l|}{ Sex } \\
\hline Male & $606(61.4)$ \\
\hline Female & $381(38.6)$ \\
\hline \multicolumn{2}{|l|}{ Age (years) } \\
\hline$<45$ & $284(28.8)$ \\
\hline $45-60$ & $424(43.0)$ \\
\hline$>60$ & 279 (28.3) \\
\hline \multicolumn{2}{|l|}{ BMI } \\
\hline $24-28 \mathrm{~kg} / \mathrm{m}^{2}$ & 249 (25.2) \\
\hline$>28 \mathrm{~kg} / \mathrm{m}^{2}$ & $738(74.8)$ \\
\hline \multicolumn{2}{|l|}{ Central obesity } \\
\hline Yes & $872(88.3)$ \\
\hline No & $115(11.7)$ \\
\hline \multicolumn{2}{|c|}{ High blood pressure } \\
\hline Yes & $481(48.7)$ \\
\hline No & $506(51.3)$ \\
\hline \multicolumn{2}{|c|}{$\begin{array}{l}\text { The course of the disease } \\
\text { (years) }\end{array}$} \\
\hline$<5$ & $308(31.2)$ \\
\hline $5-10$ & $252(25.5)$ \\
\hline $10-15$ & $204(20.7)$ \\
\hline$>15$ & 223 (22.6) \\
\hline \multicolumn{2}{|l|}{$\begin{array}{l}\text { Microvascular } \\
\text { complication }\end{array}$} \\
\hline Yes & $561(56.8)$ \\
\hline No & $426(43.2)$ \\
\hline \multicolumn{2}{|l|}{$\begin{array}{l}\text { Macrovascular } \\
\text { complication }\end{array}$} \\
\hline Yes & $552(55.9)$ \\
\hline No & $435(44.1)$ \\
\hline \multicolumn{2}{|l|}{ Smoking history } \\
\hline Yes & 565 (57.2) \\
\hline No & $422(42.8)$ \\
\hline
\end{tabular}

Table 3 (continued)

\section{Wang et al. Cost-effectiveness analysis of CCD program in Tianjin}

Table 3 (continued)

\begin{tabular}{|c|c|}
\hline Characteristics & $\mathrm{n}(\%)$ or mean (IQR) \\
\hline \multicolumn{2}{|l|}{ Drinking history } \\
\hline Yes & $357(36.2)$ \\
\hline No & $630(63.8)$ \\
\hline \multicolumn{2}{|c|}{ Family history of diabetes } \\
\hline Yes & $641(64.9)$ \\
\hline No & $346(35.1)$ \\
\hline \multicolumn{2}{|c|}{ Family history of obesity } \\
\hline Yes & $335(33.9)$ \\
\hline No & $652(66.1)$ \\
\hline \multicolumn{2}{|l|}{$\begin{array}{l}\text { Family history of } \\
\text { hypertension }\end{array}$} \\
\hline Yes & $450(45.6)$ \\
\hline No & $537(54.4)$ \\
\hline \multicolumn{2}{|c|}{$\begin{array}{l}\text { Family history of coronary } \\
\text { heart disease }\end{array}$} \\
\hline Yes & $161(16.3)$ \\
\hline No & $826(83.7)$ \\
\hline \multicolumn{2}{|c|}{$\begin{array}{l}\text { Family history of } \\
\text { cerebrovascular disease }\end{array}$} \\
\hline Yes & $86(8.7)$ \\
\hline No & 901 (91.3) \\
\hline \multicolumn{2}{|c|}{$\begin{array}{l}\text { Family history of fatty liver } \\
\text { disease }\end{array}$} \\
\hline Yes & $115(11.7)$ \\
\hline No & $872(88.3)$ \\
\hline \multicolumn{2}{|l|}{$\begin{array}{l}\text { Family history of } \\
\text { dyslipidemia }\end{array}$} \\
\hline Yes & $118(12.0)$ \\
\hline No & $869(88.0)$ \\
\hline $\mathrm{FPG}(\mathrm{mmol} / \mathrm{L})$ & $8.6(7.5,10.1)$ \\
\hline $2 \mathrm{~h} \mathrm{PG}(\mathrm{mmol} / \mathrm{L})$ & $11.7(9.8,13.7)$ \\
\hline HbA1c (\%) & $8.5(7.7,9.6)$ \\
\hline
\end{tabular}

FPG, fasting plasma glucose; PG, plasma glucose. 
Table 4 The cost-utility analyses of different sexes and ages

\begin{tabular}{|c|c|c|c|c|c|}
\hline Variable & Cost (RMB) & Incremental costs & Effectiveness (QALY) & Incremental effectiveness & ICER \\
\hline$<45$ years & $62,001.569$ & Ref & 4.333 & Ref & Ref \\
\hline $45-60$ years & $58,802.931$ & $-3,198.638$ & 4.271 & -0.0062 & $51,590.94$ \\
\hline$>60$ years & $66,487.315$ & $4,485.746$ & 4.157 & -0.176 & $-25,487.19$ \\
\hline \multicolumn{6}{|l|}{ Sex } \\
\hline Female & $56,501.834$ & Ref & 4.216 & Ref & Ref \\
\hline Male & $62,120.222$ & $5,618.388$ & 4.270 & 0.054 & $104,044.22$ \\
\hline
\end{tabular}

QALY, quality-adjusted life year; ICER, incremental cost-effectiveness ratios.

Table 5 The cost-utility analyses of each subgroup

\begin{tabular}{lccc}
\hline Variable & $<45$ years & $45-60$ years & $>60$ years \\
\hline Female & & & $54,748.2$ \\
Cost (RMB) & $59,030.9$ & $-4,282.7$ & -766.5 \\
Incremental costs & Ref & 4.272 & 4.109 \\
Effectiveness (QALY) & 4.342 & -0.07 & -0.233 \\
Incremental effectiveness & 0.07 & $61,181.49$ & $3,289.70$ \\
ICER & Ref & & $74,097.5$ \\
Male & & $58,231.8$ & $11,819.2$ \\
Cost (RMB) & $62,278.3$ & $-4,046.5$ & 4.200 \\
Incremental costs & Ref & 4.263 & -0.132 \\
Effectiveness (QALY) & 4.332 & -0.069 & $-89,539.39$ \\
Incremental effectiveness & Ref & $58,644.93$ & \\
ICER & Ref & & \\
\hline
\end{tabular}

QALY, quality-adjusted life year; ICER, incremental cost-effectiveness ratios.

elderly male group compared with the younger group. The difference in costs and QALYs gained of the elderly group $v s$. the younger group was RMB 74,097.5 vs. RMB 62,278.3, and 4.200 QALYs gained vs. 4.332 QALYs gained, respectively.

\section{Sensitivity analysis}

After removing the discounting effect and costs, compared with the female group, the comprehensive intervention strategy resulted in an increase in 0.056 QALYs and an increase in 5,747.81 RMB. Compared with the younger and elder age group, the middle-aged group was the group with the highest health gained from the comprehensive intervention program, and the associated ICER was $5,1262.33$ per QALY gained. When a $6 \%$ discounting rate of both QALYs and costs was applied in the male and female groups, the program resulted in an increase of 0.054 for QALY, and the incremental costs were 5,548.25 RMB in the male group. Furthermore, the group with the most health gained changed from the middle-aged group to the younger group, and the associated ICER was 47,789.70 per QALY gained. When a 10 -year time horizon was applied in the model, the intervention did not have a huge effect on the ICER. When a $\pm 10 \%$ of the cost was applied in each group, compared with the female group, these values leads to ICERs ranging from 93,641.28 RMB/QALY to 
114,447.17 RMB/QALY. In all age groups, compared with the middle and elderly age groups, ICERs ranged from $46,433.03$ to $56,886.68 \mathrm{RMB} / \mathrm{QALY}$. From the results of sensitivity analysis, the ICER was more sensitive to the cost variable.

\section{Discussion}

The study was limited to urban a setting since the acceleration of urbanization in China has led to the higher obesity rate and diabetes rate of these residents far more than rural residents. Moreover, given the high mobility of the rural population, it is difficult to obtain high-quality data in a rural context. This program will conduct a oneyear comprehensive intervention for participants, so the selected participants are those who have lived in Tianjin for three years or more, thus ensuring high-quality follow-up data. Finally, the results of this study could be generalized to other urban areas at the provincial level. If GDP per capita is used to measure the economic development level of cities, while life expectancy is used to measure the health status of the population, the results of this study may only be applicable to Beijing, Shanghai, and Jiangsu province. The older the patient at the start of the program, the lower the QALYs gained because older people have a poorer physical condition.

\section{Strengths}

Firstly, unlike most economic evaluation studies using simulated data by adopting Monte Carlo methods, the data from this study was derived from a real-world setting thus making the results more reliable. The real-world data is not only reflected in the actual results of each patient's physical examination but also includes the cost calculated according to the specific medication situation of each patient and the inspection items. The transportation expenses and communication expenses are calculated according to local living standards. Additionally, after participant recruitment, patients with different glycated hemoglobin levels will provide an initial probability to the model. After three months of comprehensive intervention, the glycosylated hemoglobin changed in these patients. According to these changes, we obtained the transfer probability matrix. The transition probability of each cycle after three months was obtained according to the preset formula in our model, while most cost-effectiveness analysis using the Markov models derive transfer probabilities from data simulations.
Second, this program is aimed at overweight and obese patients with T2DM. As a growing number of people with T2DM, no other studies have examined the costeffectiveness of a comprehensive intervention program for this population except for this study. Finally, prediabetic patients were not recruited because not all prediabetic patients will acquire diabetes. The definition of "prediabetes" will make people who are essentially healthy become patients with excessive pressure. This pressure is unnecessary, and on the contrary, the pressure itself will increase the occurrence of diabetes. Diabetes can and should be prevented, but we do not need every healthy person to be a patient. In this study, effective intervention for patients with T2DM was considered to have more practical significance.

\section{Weaknesses}

Because patients with severe diabetic complications were excluded, the health utility values might have been overestimated. Moreover, since the study did not include a control group that received only standard care, the costeffectiveness analysis of the comprehensive intervention compared to standard care could not be performed. However, our study aimed to evaluate the cost-effectiveness within the CCD program for different age and gender groups, and not versus "standard care".

\section{Conclusions}

The results of the economic evaluation study suggest that a comprehensive intervention program for overweight and obese type 2 diabetes patients of different ages and genders is likely to be cost-effective in Tianjin, and that a maximum WTP for a QALY gain of threefold the GDP per capita should be treated as the decision-making criteria. When running the model with different scenarios of age and gender group, the model results showed that after participating in this program, men spent more money than women; however, men also gained higher QALYs. If $356.8 \mathrm{~K} \mathrm{RMB}$ per QALY gained is assumed to be the threshold of cost-effectiveness, then this program is more cost-effective in the male group than in the female group over a 5 -year period. In the all-ages male group, compared with the younger group, the results were more favorable for the middle-aged group with a positive ICER value; the intervention has economic benefits for this group if the WTP is adopted. In the all-ages female group, compared 
with the younger group, the ICERs of the middle and elderly age groups were both positive. The ICER was more favorable for the elderly women group. The results indicate that the program has the potential to be cost-effective, but a definitive conclusion based on this study cannot be made, as it is an interim analysis.

\section{Acknowledgments}

We would like to thank Novo Nordisk for providing data and for assisting in coordinating our cooperation with each hospital involved in this program. We would also like to express our appreciation for the assistance we received from each professor and nurse from these hospitals.

Funding: Financial support came from The Ministry of Education of the Humanities and Social Science Project (grant No. 17YJAZH048), Key Projects of Tianjin Science and Technology Commission in 2016 (No.16ZXMJSY00150), and the National Natural Science Foundation of China (grant No. 81803333). The study was funded by Novo Nordisk (China) Pharmaceuticals Co. (Award No. SD20150204), http://www. novonordisk.com.cn/.

\section{Footnote}

Conflicts of Interest: The authors have no conflicts of interest to declare.

Ethical Statement: The authors are accountable for all aspects of the work in ensuring that questions related to the accuracy or integrity of any part of the work are appropriately investigated and resolved. The protocol of this study was approved by the Institutional Review Board of Tianjin Medical University. All participants signed informed consent before participation in the study.

\section{References}

1. Shi XD, He SM, Tao YC, et al. Prevalence of obesity and associated risk factors in Northeastern China. Diabetes Res Clin Pract 2011;91:389-94.

2. Yang W, Lu J, Weng J, et al. Prevalence of Diabetes among Men and Women in China. N Engl J Med 2010;362:1090-101.

3. Schwartz S, Fabricatore AN, Diamond A. Weight reduction in diabetes. Adv Exp Med Biol 2012;771:438-58.

4. International Diabetes Federation. IDF Diabetes Atlas [EB/ OL]. (2017) [2018-12-19]. Available online: http://www. diabetesatlas.org/

5. World Health Organization. Global Health Observatory data repository, Probability of dying per 1000 live births. [EB/OL]. (2016-04) [2018-12-20]. Available online: http:// apps.who.int/gho/data/node.main.525?lang=en

6. World Health Organization. Global Health Observatory data repository, life table by country. [EB/OL]. (2016-04) [2018-12-20]. Available online: http://apps.who.int/gho/ data/node.main.LIFECOUNTRY?lang=en

7. Melmed S, Polonsky KS, Larsen PR, et al. Williams textbook of endocrinology. 12th ed. USA: Elsevier/ Saunders, 2011.

8. World Health Organization. Global report on diabetes: Managing diabetes [EB/OL]. (2016-04) [2018-12-20]. Available online: http://www.who.int/diabetes/globalreport/en/

9. Pelletier C, Dai S, Roberts KC, et al. Diabetes in Canada: facts and figures from a public health perspective. Chronic Dis Inj Can 2012;33:53-4.

10. Gaede P, Lund-Andersen H, Parving HH, et al. Effect of a multifactorial intervention on mortality in type 2 diabetes. N Engl J Med 2008;358:580-91.

11. Effect of intensive blood-glucose control with metformin on complications in overweight patients with type 2 diabetes (UKPDS 34). UK Prospective Diabetes Study (UKPDS) Group. Lancet 1998;352:854-65.

12. Clarke P, Gray A, Adler A, et al. Cost-effectiveness analysis of intensive blood-glucose control with metformin in overweight patients with type ii diabetes (UKPDS No. 51). Diabetologia 2001;44:298-304.

13. Xie X, Vondeling H. Cost-utility analysis of intensive blood glucose control with metformin versus usual care in overweight type 2 diabetes mellitus patients in Beijing, P.R. China. Value Health 2008;11:S23-32.

14. Saha S, Leijon $M$, Gerdtham U, et al. A culturally adapted lifestyle intervention addressing a Middle Eastern immigrant population at risk of diabetes, the MEDIM (impact of Migration and Ethnicity on Diabetes In Malmö): study protocol for a randomized controlled trial. Trials 2013;14:279.

15. Neumann A, Lindholm L, Norberg M, et al. The costeffectiveness of interventions targeting lifestyle change for the prevention of diabetes in a Swedish primary care and community-based prevention program. Eur J Health Econ 2017;18:905-19.

16. Neumann A, Schwarz P, Lindholm L. Estimating the cost-effectiveness of lifestyle intervention programmes 
to prevent diabetes based on an example from Germany: Markov modelling. Cost Eff Resour Alloc 2011;9:17.

17. The Diabetes Prevention Program Research Group. Within-trial cost-effectiveness of lifestyle intervention or metformin for the primary prevention of type 2 diabetes. Diabetes Care 2003;26:2518-23.

18. Briggs A, Sculpher M. An introduction to markov modelling for economic evaluation. Pharmacoeconomics 1998;13:397-409.

19. Gagniuc PA. Markov Chains: From Theory to Implementation and Experimentation. Wiley, 2017.

20. Welsing PM, Severens JL, Hartman M, et al. The initial validation of a Markov model for the economic evaluation of (new) treatments for rheumatoid arthritis. Pharmacoeconomics 2006;24:1011-20.

Cite this article as: Wang $\mathrm{H}$, Wang $M$, Wang $\mathrm{J}$, Liu $\mathrm{H}, \mathrm{Lu}$ R, Duan T, Gong X, Feng S, Cui Z, Liu Y, Li C, Ma J. Costeffectiveness analysis of comprehensive intervention programs to control blood glucose in overweight and obese type 2 diabetes mellitus patients based on a real-world setting: Markov modeling. Ann Transl Med 2019;7(22):676. doi: 10.21037/ atm.2019.10.38
21. Sopina E, Martikainen JA, Spackman E, et al. Validation of a Markov model for economic evaluation of screening and preventive interventions in Alzheimer's disease in Denmark. Value in Health 2015;18:A695.

22. Liu X, Li C, Gong H, et al. An economic evaluation for prevention of diabetes mellitus in a developing country: a modelling study. BMC Public Health 2013;13:729.

23. Garber AJ, Abrahamson MJ, Barzilay JI, et al. Consensus statement by the American Association Of Clinical Endocrinologists and American College of Endocrinology on the comprehensive type 2 diabetes management algorithm - 2019 executive summary. Endocr Pract 2019;25:69-100.

24. Pan CW, Sun HP, Wang X, et al. The EQ-5D-5L index score is more discriminative than the EQ-5D-3L index score in diabetes patients. Qual Life Res 2015;24:1767-74. 
Supplementary

Table S1 Intervention costs

\begin{tabular}{lc}
\hline Variables & Costs (RMB) \\
\hline Direct medical cost (per person) & 228 \\
Screening fee & $(2 \times 44.00)=88.00$ \\
Blood lipids & $(2 \times 50.00)=100.00$ \\
Glycated hemoglobin & $(4 \times 10.00)=40.00$ \\
Glucose tolerance & $3,234.16$ \\
Drugs (mean) & \\
Indirect medical cost (per person) & $(8 \times 12.60)=100.80$ \\
Transportation costs & 6.25 \\
Phone hotline & \\
Summary (per person) & $3,462.16$ \\
Direct medical cost & 107.05 \\
Indirect medical cost & $3,569.21$ \\
Total costs of 3 months &
\end{tabular}

